\title{
Phase Behavior of Pseudo-Ternary Gemini Surfactant + 1-Hexanol/Oil/Water Systems
}

\author{
Mohd Zul Helmi Rozaini ${ }^{1,2}$ \\ ${ }^{1}$ School of Environmental Sciences, University of East Anglia, Norfolk, Norwich NR4 7TJ, UK \\ ${ }^{2}$ Department of Chemical Sciences, University Malaysia Terengganu, Terengganu 21030 Kuala, Terengganu, Malaysia
}

Correspondence should be addressed to Mohd Zul Helmi Rozaini, zulhelmi@umt.edu.my

Received 3 July 2012; Accepted 7 October 2012

Academic Editor: Ali Hussain Reshak

Copyright (C) 2012 Mohd Zul Helmi Rozaini. This is an open access article distributed under the Creative Commons Attribution License, which permits unrestricted use, distribution, and reproduction in any medium, provided the original work is properly cited.

\begin{abstract}
Temperature dependent phase behavior of pseudo-ternary Gemini surfactant +1 -hexanol (1:5 molar ratios)/oil/water systems is reported from $0^{\circ} \mathrm{C}$ to $65^{\circ} \mathrm{C}$. The influence of nature of hydrocarbon oil and type of electrolytes (weak as well as strong) has been investigated on the temperature induced phase behavior of the ternary system. At surfactant concentration, $\Phi s=40 \%$, a "nose-shaped" microemulsion region is observed. Below one-phase microemulsion region, $L_{\alpha}$ phase appears. The presence of $\mathrm{NaCl}$ decreases the domain size of $1 \Phi$ micellar region whereas oxalic acid first decreases the domain below $\Phi_{w}<18$ and then increases above $\Phi_{w}>18$ in the lower boundary of the phase diagram. The critical weight fraction of water $\Phi_{w}{ }^{\text {cri }}$ decreases in presence of both electrolytes. However, $\Phi_{w}{ }^{m a x}$ increases in presence of oxalic acid and remains constant in presence of $\mathrm{NaCl}$ as compared to salt-free system. Furthermore, when cyclohexane was replaced by a longer straight chain hydrocarbon, dodecane, the domain of the one-phase microemulsion region is tremendously increased.
\end{abstract}

\section{Introduction}

Microemulsions (MEs) are optically transparent, thermodynamically stable, nanostructured mixture of oil and water stabilized by surfactant and cosurfactant [1]. Microemulsions have attracted great interest because of their unique physiochemical characteristics such as large stabilization capacity, ultralow interfacial tension, and a very large interfacial region, and because of their potential industrial applications such as enhanced oil recovery, biotechnology, nanotechnology, novel drug delivery, agriculture, beverages, and chemical reaction [2].

Phase behavior and structural organization of microemulsions are known to play key roles in its industrial and technological applications. Phase behavior studies provide information on the phase boundaries of different phases as a function of composition and temperature and more important structural organization of surfactant aggregates can also be inferred. In addition, it allows comparison of efficiency of different surfactant for a given application. The boundaries of one-phase micellar region can be easily accessed by visual observation of the sample of known composition. However, long equilibration is required in multiphase regions especially if a liquid crystalline phase is involved and this makes phase determination tedious, time consuming, and difficult [1-3] to construct.

The phase behavior and structural organization of surfactant aggregates are highly dependent on the elastic properties of surfactant monolayer separating oil and water domains [4]. The natural curvature of the surfactant monolayer depends upon the geometric properties of the hydrophilic and hydrophobic moiety of the surfactant molecules [3]. These properties, in turn, depend on the temperature, pressure, ionic strength of water, nature of oil, and so forth. For a given surfactant, a change in the natural curvature from water (positive) to oil (negative) or vise versa can be achieved by changing one or more formulation variables. The most pronounced effect is temperature for nonionic surfactant and salts for ionic surfactant $[4,5]$.

This phase behavior of a ternary system is often studied as a function of temperature and composition and the position of different phases are determined within the phase prism 
by erecting a vertical section of the prism at constant surfactant concentration. The phase boundaries give rise to characteristic "channel" shown in Figure 1(a), frequently reported by Rozaini and Brimblecombe [6]. For a ternary system water (A), Oil (B), and surfactant (C), at constant pressure the ternary system is specified by setting three independent variables. The three most common variables are temperature $T$, weight percentage $\Phi$ or volume fraction $\alpha$ of the oil, either of which is given by $\mathrm{B} /(\mathrm{A}+\mathrm{B})$ in the mixture of oil and water, and the weight fraction of the surfactant in the mixture, $\gamma=\mathrm{C} /(\mathrm{A}+\mathrm{B}+\mathrm{C})$. Each point in the threedimensional phase space of temperature and composition is then defined by giving variables $T, \Phi$ or $\alpha$, and $\gamma$. It is easiest to discuss phase behavior if it is expressed in an upright triangle A-B-C as the base (Figure $1(\mathrm{a})$ ), but now temperature is the field variable. This is done by mixing a known amount of surfactant in oil and making surfactant concentration constant and water is added gradually to increase $\alpha$ or $\Phi$ value from $0 \%$ to $100 \%$. Several samples having $\gamma$ constant are made. For each sample, temperature is raised and the phase sequence 2-1-2 is observed with increase in temperature. Plotting the result yields the phase diagram shown in Figure 1(a) and also shown in Figure 1(b) in the vertical section of the phase prism at constant $\gamma$. The shape of the phase diagram obtained on such section looks like a "channel." The one-phase "channel" is sandwiched between two two-phase regions. The size and shape of the "channel" and the temperature at which it is located provide key information about the system. At lower temperature, the nonionic amphiphile is better soluble in water than in oil, and o/w microemulsion is formed which equilibrates with excess oil phase (signified by 2). At higher temperature, the nonionic surfactant is more oil soluble than water, and w/o microemulsion is formed in equilibrium with excess aqueous phase (signified by 2). This is due to the fact that the solubilization of the nonionic amphiphile in water is driven by the formation of the hydrogen bonds [3]. These hydrogen bonds break with rising temperature, causing a better oil solubility of the amphiphile. Alternatively, nonionic surfactant becomes hydrophobic with rise in temperature due to conformational changes in the polyoxyethylene chain of the nonionic Gemini surfactant. As a result, solubility of the surfactant increases in hydrocarbon oil with rise in temperature $[3,4]$.

Furthermore, nonionic microemulsions are of increasing importance in several industrial applications. They are highly used in liquid detergent formulations [5]. Nonionic Gemini surfactants are more biodegradable than their ionic counterparts and more suitable for applications in cosmetic, foods, and personal care products [7]. It is known that the control of phase state is very important in several applications [8] and this prompted to investigate the temperature dependent phase behavior of microemulsions of a well known nonionic surfactant, Gemini surfactant which has been used for various chemical reactions [9]. In this context, in this paper temperature dependent phase behavior of pseudo-ternary Gemini + 1-Hexanol/oil/water systems is reported over a wide range of temperature and composition. The influence of nature of hydrocarbon oil and type of electrolytes (weak

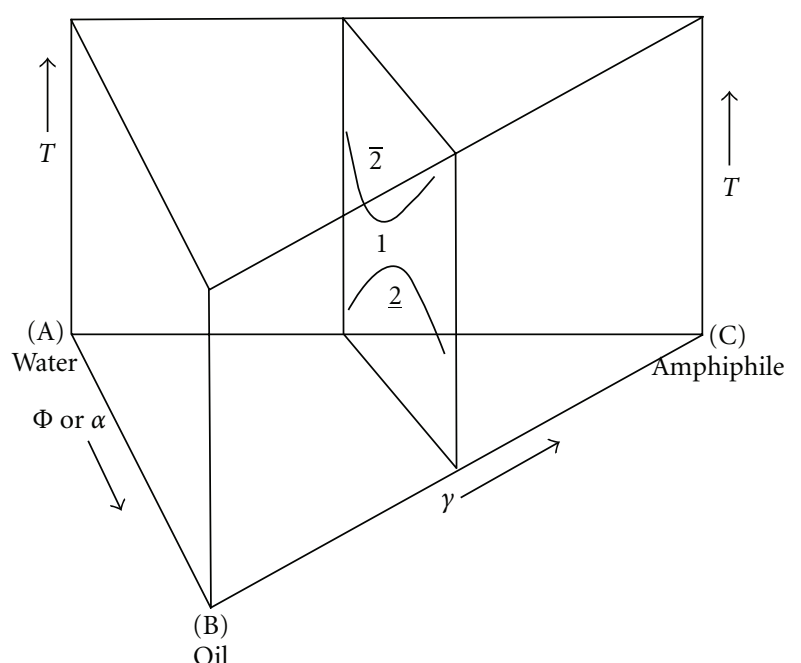

(a)

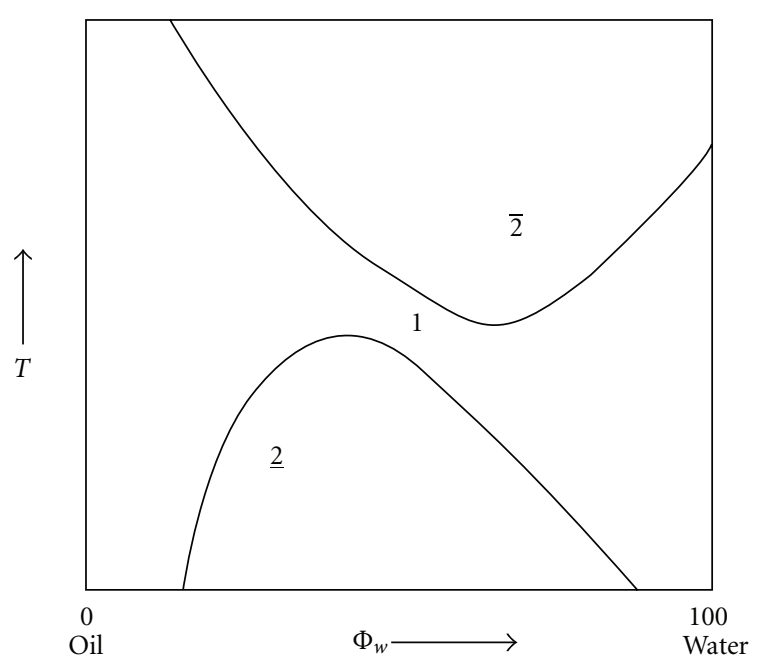

(b)

Figure 1: (a) Schematic "channel-shaped" phase prism of a ternary mixture of oil/water/nonionic surfactant in temperaturecomposition space at constant concentration $\gamma$ showing a single phase (1Ф) and two two-phase regions (b) Schematic "channelshaped" phase diagram of a ternary mixture of oil/water/nonionic surfactant at vertical section of the phase prism at constant surfactant concentration $\gamma$. The single phase $(1 \Phi)$ is surrounded by two two-phase regions in which nonionic Gemini surfactant is dissolved either aqueous bottom phase (2) or organic top phase (2). For detail please see text.

as well strong) was investigated on the temperature induced phase behavior of the ternary system. Crossed Polaroid microscope was used to distinguish between one-phase isotropic micellar region and anisotropic liquid crystalline or turbid phase.

\section{Experimental}

2.1. Materials. The nonionic Gemini (Disodium dicocamide PEG-15) surfactant was obtained from Sigma Aldrich, 
Malaysia. Anhydrous dodecane and 1-hexanol were procured from Schuchardt, Germany. Sodium chloride (AR) was purchased from Fisher Scientific, Singapore. Oxalic acid (AR) was purchased from Sigma Aldrich, Malaysia. Double distilled water was used throughout the experiment.

\subsection{Methods}

2.2.1. Constructing a Pseudo-Ternary Phase Diagram. Phase diagram of Gemini surfactant + 1-hexanol (1:5 molar ratio)/ oil/water or aqueous electrolyte systems were constructed by titration method and the phase boundaries of one-phase micellar region of the ternary systems were determined at fixed surfactant concentration, $\gamma=40 \%$. A known amount of surfactant + cosurfactant and oil is taken and subsequently the mixture was titrated with water or aqueous electrolyte solution with the help of Hamilton microsyringe. The final mixture was gently stirred and then immersed in a temperature controlled water bath to equilibrate different phases at desired temperature. Characterization of the resulting isotropic one-phase micellar region or anisotropic phases was done visually and through crossed Polaroid microscope after allowing sufficient time (typically 4-6 hours) for attainment of equilibrium at a particular temperature. The entire phase diagram was mapped in this manner. The detailed procedure for the determination of the phase diagram has been described elsewhere.

\section{Results and Discussions}

One of the most common and convenient method to study the phase behavior and related properties of a ternary system, surfactant/oil/water, is to characterize the system at the vertical section of the prism at fixed amphiphile concentration. In this respect, phase behavior of pseudo-ternary Gemini surfactant + 1-hexanol (1:5 molar ratio)/cyclohexane/water system was determined as a function of temperature $T$ and weight fraction of water $\Phi_{w}$ and shown in Figure 2. The weight fraction of surfactant $\gamma$ was kept constant and equal to $40 \%$. It is evident from the figure that the domain of one-phase ME displays a "nose-shaped" region. Similar "nose-shaped" micellar regions were observed by Jeong and coworkers [9], and Rozaini and Brimblecombe [10] for nonionic Gemini surfactant/oil/water ternary systems.

It is evident from Figure 2 that at $\Phi_{w}<5$, the temperature window for one-phase micellar region is from below $0^{\circ} \mathrm{C}$ to above $60^{\circ} \mathrm{C}$. At $\Phi_{w}>5$, the upper boundary of micellar phase decreases with increase in water content. At $\Phi_{w} \approx 0-15, L_{\alpha}$ phase or two-phase region (2) does not exit even below $0^{\circ} \mathrm{C}$. At $\Phi_{w}>15, L_{\alpha}$ starts to appear in the lower portion of the phase diagram, and, therefore, the weight fraction of water $\left(\Phi_{w} \approx 0-15\right)$ may be defined as the critical weight fraction of water $\Phi_{w}{ }^{\text {cri }}$ where phase transition occurs from $1 \Phi$ micellar region to $L_{\alpha}$ phase. At $\Phi_{w} \geq 15$, with rise in temperature, transition from $L_{\alpha}$ to one-phase ME region takes place and the transition is very sharp $\left(0.1^{\circ} \mathrm{C}\right)$ without formation of two-phase system. With further increase in temperature, one-phase ME region changes into two-phase region. Below one-phase micellar

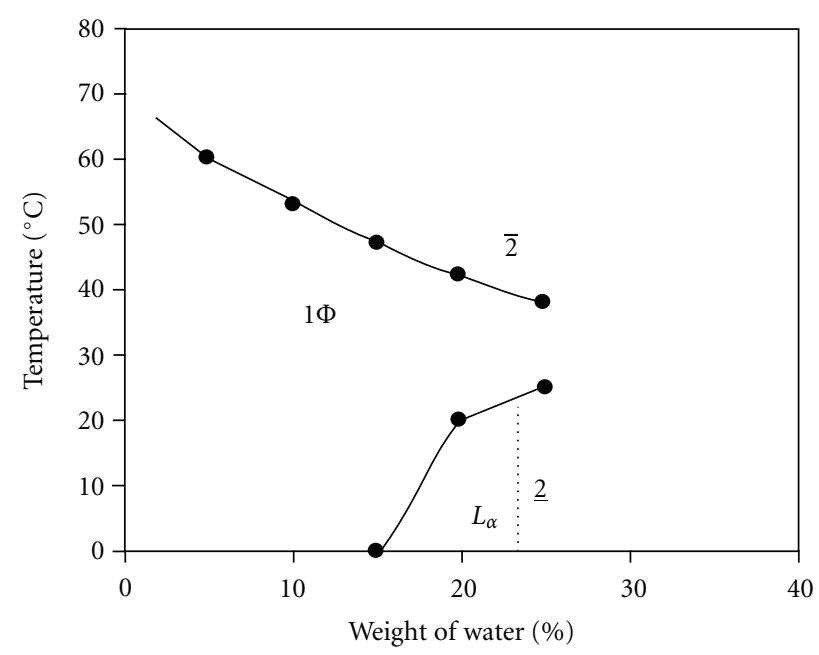

FIgUre 2: Temperature dependent phase diagram of Gemini surfactant +1 -hexanol/cyclohexane/water at surfactant concentration $(\gamma=40 \%)$ showing a single phase microemulsion region and $L_{\alpha}$ phase.

region, the domain of $L_{\alpha}$ increases with increase in weight fraction of water and then finally changes into two-phase region (2). The exact boundary of the $L_{\alpha}$ phase was not determined in this set of experiments. One-phase micellar region is present up to $\Phi_{w} \approx 25$ and this may be defined as $\Phi_{\max }$ beyond which one-phase ME does not appear at any temperature and composition and only two-phase region was observed. The one-phase micellar region does not touch water axis. It means that $\Phi_{s} \approx 40 \%$; Gemini-hexanol is unable to form normal micelle at any temperature in between $0^{\circ} \mathrm{C}$ and $60^{\circ} \mathrm{C}$ and also does not form oil swollen micelles (o/w microemulsions) near water axis. Otherwise one-phase micellar region must be extended up to water axis and one could expect a broad spectrum of micellar region in place of two-phase region beyond $\Phi_{w}>25$ in the ternary phase diagram. Further, at $\Phi_{w} \approx 0-5$, the upper boundary of ME region is above $60^{\circ} \mathrm{C}$. With increase in weight fraction of water $\left(\Phi_{w}\right)$, the upper boundry of temperature window of $\mathrm{ME}$ region decreases. As a result, a narrower temperature span of the ME region is observed and the domain of ME looks "nose-shaped."

Furthermore, it is known that the temperature dependent phase behavior of nonionic surfactant/oil/water system arises in the first place from the variation of local concentration of water in the ethylene glycol layer, which modulates the surfactant film curvature. But it also originates from the solubility variation of the surfactant monolayer in the organic phase occurring as a function of temperature $[11,12]$. The phase diagram for the system, Gemini surfactant +1 -hexanol/cyclohexane/water, plotted in Figure 2 corresponds to the oil rich region of the prism diagram. The shape and size of the ME domain in Figure 2 give the key information about the ternary system. At $\Phi_{w}<5$, in the lower boundary, the one-phase micellar region is hydrated reversed micelles. At $\Phi_{w} \geq 15$, Gemini +1 -hexanol forms lamellar structure resulting into $L_{\alpha}$ phase. With increase in 
temperature $L_{\alpha}$ phase turns into one-phase ME. With further increase in temperature, one-phase micellar system changes into two-phase region (2) in which water swollen reverse micelles equilibrates with the excess aqueous phase.

Researchers had studied the temperature dependent phase behavior of C12E4/dodecane/water and observed similar "nose-shaped" micellar region [13]. However, critical weight fraction of water $\Phi_{w}{ }^{\text {cri }}$ and maximum weight fraction of water, $\Phi_{w}^{\max }$ were 10 and 56 , respectively. $L_{\alpha}$ phase was also observed below one-phase microemulsion region in the lower boundary of the phase diagram. In the present studies, $\Phi_{w}{ }^{\text {cri }}$ is equal to $\approx 15$, and $\Phi_{w}{ }^{\max }$ is equal to $\approx 25$. By comparing these results, it is evident that the temperature dependent phase behavior of nonionic surfactant/oil/water is highly dependent on the amphiphilicity (hydrocarbon chain length) of the surfactant. If the amphiphilicity is decreased, one-phase ME region shrinks. That is what observed in the present investigations.

3.1. Influence of Electrolytes. The influence of $0.1 \mathrm{M} \mathrm{NaCl}$ and $0.3 \mathrm{M}$ oxalic acid on the temperature induced phase behavior of the system, Gemini surfactant + 1-hexanol/ cyclohexane/water, was investigated and shown in Figure 3. In presence of both electrolytes, domain of $1 \Phi$ micellar phase display "nose-shaped" regions similar to the salt free system. However, the presence of electrolytes changes the phase boundaries of the one-phase micellar region. It is evident from the Figure 3 that the critical weight fraction of water $\Phi_{w}{ }^{\text {cri }}$ decreases in presence of both electrolytes, strong as well as weak. The critical weight fraction of water $\Phi_{w}{ }^{\text {cri }}$ is equal to 10 in presence of both electrolytes whereas it is 15 for salt free system. However, $\Phi_{w}{ }^{\max }$ increases in presence of oxalic acid and there is no change in $\Phi_{w}{ }^{\max }$ in presence of $\mathrm{NaCl}$ as compared to salt free system. At $\Phi_{w} \approx 5$, the temperature window for one-phase micellar region is from below $0^{\circ}-54^{\circ} \mathrm{C}$ in presence of oxalic acid, from below $0^{\circ}-$ $50^{\circ} \mathrm{C}$ in presence of $\mathrm{NaCl}$, and from below $0^{\circ}-60^{\circ} \mathrm{C}$ for salt free system; that is, the presence of electrolytes contracts the temperature window of one-phase micellar region but the contraction is more in presence of strong electrolyte $(\mathrm{NaCl})$ than the weak one (oxalic acid).

In the lower boundary, at $\Phi_{w}>10$, the presence of $\mathrm{NaCl}$ and oxalic acid has opposite effect on the domain size of the micellar region. In the lower boundary, strong electrolyte, $\mathrm{NaCl}$, always decreases domain of the $1 \Phi$ microemulsion region whereas oxalic acid first decreases the domain of $1 \Phi$ micellar region below $\Phi_{w}<18$ and then increases the same above $\Phi_{w}>18$. In the upper boundary, the presence of oxalic acid expands temperature window of $1 \Phi$ micellar region above $\Phi_{w}>14$ and the presence of $\mathrm{NaCl}$ increases the same at $\Phi_{w}>19$. Thus the presence of weak electrolyte, oxalic acid, expands $1 \Phi$ micellar region more as compared to $\mathrm{NaCl}$ in the upper region of the phase diagram. As a result, a bigger domain $1 \Phi \mathrm{ME}$ region is observed at higher water content.

The changes in the phase boundaries of one-phase micellar region (Figure 3) arises due to the presence of electrolytes in the aqueous domain of the ternary system. It is known that the phase behavior of a ternary system is highly influenced by the presence of electrolytes [14-16].

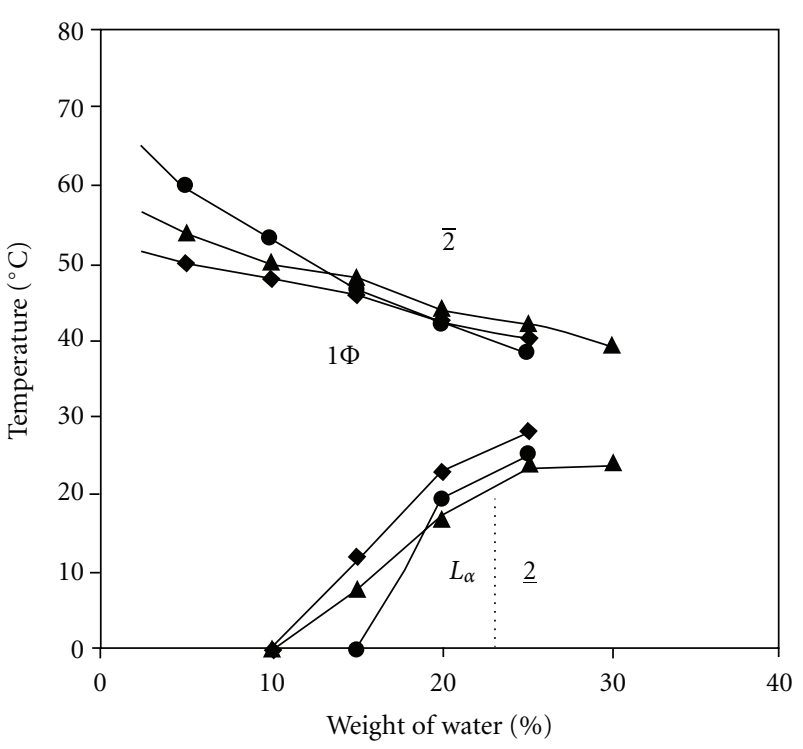

Figure 3: Temperature dependent phase diagram of Gemini Surfactant +1 -hexanol/cyclohexane/water at surfactant concentration $(\gamma=40 \%)$ in presence of $0.1 \mathrm{M} \mathrm{NaCl}$ (black diamond), $0.3 \mathrm{M}$ oxalic acid (black triangle), and electrolyte free (black circle).

The presence of electrolytes reduces the solubility of the hydrophilic head group of the ionic surfactant amphiphile in water. In case of nonionic amphiphiles they compete for the hydrating water of the head groups. The presence of electrolytes ( $\mathrm{NaCl}$ or oxalic acid) may decrease electrostatic interactions between the polar head groups of the surfactant and thereby coming closure to each other. This would lead more rigid interface, smaller intermicellar interaction, and decrease in size of the core of reverse micelles (RMs) and thus less amount of water is solubilized inside the core RMs in presence of electrolytes and this results in apparent decrease in $\Phi_{w}{ }^{\text {cri }}$ from 15 (salt free system) to 10 (in presence of electrolytes). Further, it can be seen from Figure 3 that at $\Phi_{w} \approx 5,1 \Phi$ micellar region has upper critical temperature $\sim 50^{\circ} \mathrm{C}$ in presence of $0.1 \mathrm{M} \mathrm{NaCl}, \sim 54^{\circ} \mathrm{C}$ in presence of $0.3 \mathrm{M}$ oxalic acid and $\sim 60^{\circ} \mathrm{C}$ for salt free system; that is, the presence of electrolytes decreases the upper critical transition temperature $T_{\text {upper }}{ }^{\text {cri }}$ and the decrease in $T_{\text {upper }}{ }^{\text {cri }}$ is much more in presence of $\mathrm{NaCl}$ than oxalic acid. It is known [6] that on the oil-rich side, w/o MEs are present at low water content near upper critical temperature (near upper boundary of the phase diagram), which coagulate with decreasing temperature.

The presence of electrolyte decreases the size of RMs of Gemini at any temperature [17] due to ion-dipole interaction or complexation between cations and polyoxyethylene chain of double headed Gemini surfactant structures that results in a closure packing of polar head groups of Gemini surfactant and decreases the extent of solubilization of aqueous electrolyte solution inside the core of RMs. As a result, one-phase micellar region changes into two-phase region in presence of electrolytes relatively at a lower temperature as compared to salt free system. This causes an apparent 
decrease in upper critical temperature $T_{\text {upper }}{ }^{\text {cri }}$ in presence of electrolyte and the decrease is more in the presence of strong electrolyte $(\mathrm{NaCl})$ than the weak one (oxalic acid). This may be due to a greater extent of ion-dipole interaction or complexation between the cations and polyoxyethylene chain of Gemini in presence of the strong electrolyte $(\mathrm{NaCl})$ as compared to the weak electrolyte (oxalic acid). As a result, the upper critical transition temperature, Tupper cri decreases much more in presence of strong electrolyte $(\mathrm{NaCl})$ than the weak one (oxalic acid) and phase transition from onephase micellar region to two-phase region occurs at relatively lower temperature $\left(\sim 50^{\circ} \mathrm{C}\right)$ in presence of $\mathrm{NaCl}$ than oxalic acid $\left(\sim 54^{\circ} \mathrm{C}\right)$. As a result, temperature window of onephase micellar region becomes narrower in presence of both electrolytes as compared to salt free system.

Furthermore, it is known that the type of ME depends upon water-to-oil ratio and if $\Phi_{w} \approx 25-70 \mathrm{wt} \%$, a spongelike structure is obtained which is known as bicontinuous ME in which surfactant monolayer separates into disordered, interpenetrating domains of oil and water. The size of the domains of water and oil in a bicontinuous ME is highly dependent on amphiphile concentration and water-to-oil ratio. In the middle of phase diagram, at $\Phi_{w}>18$, the presence of electrolytes expands the temperature window of $1 \Phi$ micellar region in the upper region of the phase diagram. This may be due to the fact that the presence of electrolytes (weak or strong) makes the surfactant (Gemini) more hydrophobic. That causes a better oil solubility of Gemini surfactant in oil domain and therefore phase transition from $1 \Phi \mathrm{ME}$ region to two-phase region takes place relatively at a higher temperature and higher water content as compared to salt free system. As a result, temperature window for one-phase micellar and $\Phi^{\max }$ increase in presence of both electrolytes. Further, at $\Phi_{w}>18$, the presence of oxalic acid expands the temperature window of $1 \Phi \mathrm{ME}$ region more than $\mathrm{NaCl}$ and this may be due the fact that oxalic acid probably increases hydrophobicity of the Gemini surfactant more than $\mathrm{NaCl}$ due to higher concentration of oxalic acid. This results in an increase in the temperature window of onephase micellar region in the middle of phase diagram.

3.2. Influence of Hydrocarbon Chain Length of Oil. The influence of the hydrocarbon chain length of oil on the temperature induced phase behavior of the ternary system. Gemini surfactant +1 -hexanol $(1: 5$ molar ratio)/oil/0.1 M $\mathrm{NaCl}$ was investigated. Cyclohexane was replaced by a longer straight chain hydrocarbon, dodecane, and domain of one-phase micellar region of Gemini Surfactant +1 hexanol/dodecane $/ 0.1 \mathrm{M} \mathrm{NaCl}$ was determined and shown in Figure 4. It is clear from the figure that the general features of one-phase micellar regions are similar in presence of both oils, that is, dodecane and cyclohexane. The critical weight fraction of water $\Phi_{w}{ }^{\text {cri }}$ is 10 in presence of cyclohexane and 15 in presence of dodecane. Also, there is a tremendous increase in $\Phi_{w}{ }^{\max }$ in presence of dodecane as compared to cyclohexane and is equal to $40 \%$ whereas it is $25 \%$ in presence of cyclohexane. At $\Phi_{w}=5$, the upper critical temperature $T_{\text {upper }}$ cri is increased and is equal to $70^{\circ} \mathrm{C}$ in

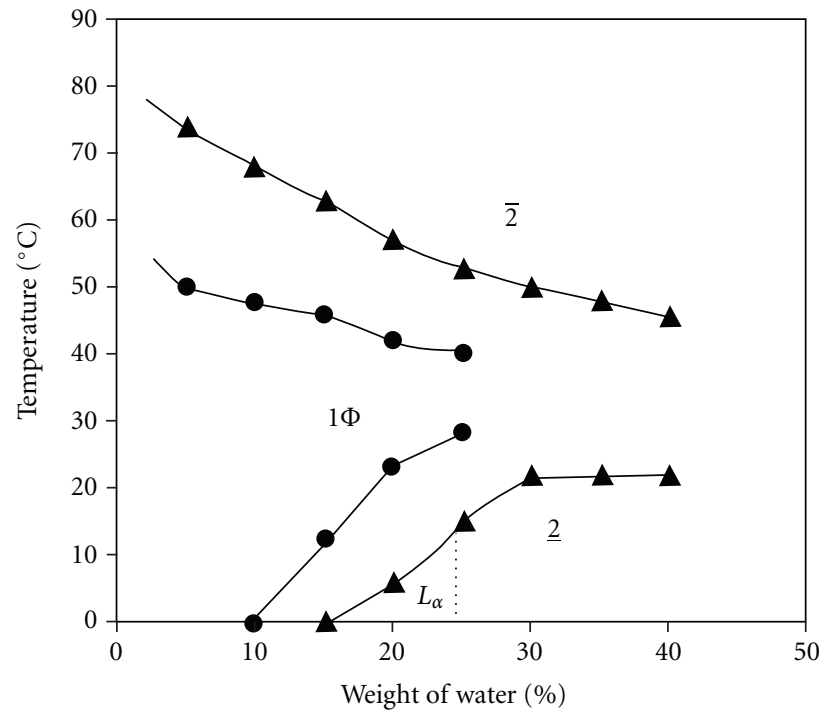

Figure 4: Temperature dependant phase diagram of Gemini surfactant + 1-hexanol/oil/0.1 M NaCl at constant surfactant concentration $(\gamma=40 \%)$ in presence of cyclo-hexane (black circle) and dodecane (black triangle).

presence of dodecane. Thus the temperature window of onephase micellar is very much larger in presence of dodecane as compared to cyclohexane. In the lower boundary of the phase diagram, three is tremendous increase in domain of onephase micellar in presence of dodecane. It is quite interesting to note that the one-phase micellar region does not touch the water axis in presence of dodecane also. It means that Gemini surfactant + 1-hexanol is unable to form normal micelles or oil swollen ME near water axis in presence of oil, dodecane, or cyclohexane.

The changes in phase boundary in one-phase micellar region occurs due to different nature of the oils used here in the presence studies. The presence of longer straight chain hydrocarbon, dodecane, tremendously increases the domain of $1 \Phi$ micellar region as compared to cyclohexane. The critical weight fraction of water $\Phi_{w}{ }^{\text {cri }}$ and $\Phi_{w}{ }^{\max }$ are larger in presence of dodecane as compared to cyclohexane. Also Tuppercri has been increased in presence of dodecane. As a result, the temperature window of one-phase micellar region increases. Thus the solubilization efficiency of the amphiphile, Gemini surfactant, is much more in presence of dodecane than cyclohexane and this may be attributed to higher hydrophobicity (longer hydrocarbon chain length) of dodecane. It is known that with rise in temperature, the nonionic amphiphile of Gemini becomes hydrophobic [1618]. As a result, its solubility increases in more hydrophobic oil, dodecane, than cyclohexane. Thus phase transition from one-phase micellar region to two-phase region takes place relatively at higher temperature. This causes an apparent increase in $T_{\text {upper }}{ }^{\text {cri }}$ in presence of dodecane as compared to cyclohexane. As a result, the temperature window of onephase micellar region becomes larger in presence of dodecane as compared to cyclohexane and the structures are also promising for nonlinear optical materials [19]. 


\section{Conclusions}

The temperature dependent phase behavior of Gemini Surfactant + 1-pentanol (1:5 molar ratio)/cyclohexane or dodecane/water or brine systems was investigated. At surfactant concentration, $\gamma_{s}=40 \%$, a "nose-shaped" microemulsion region is observed. Below one-phase micellar region liquid crystalline $L_{\alpha}$ appears. The one-phase micellar region does not touch water axis. At $\Phi_{w} \approx 5$, the temperature window for one-phase micellar region is from below $0^{\circ} \mathrm{C}-50^{\circ} \mathrm{C}$ in presence of $\mathrm{NaCl}$, below $0^{\circ} \mathrm{C}-54^{\circ} \mathrm{C}$ in presence of oxalic acid, and form below $0^{\circ} \mathrm{C}-60^{\circ} \mathrm{C}$ for salt free system in presence of cyclohexane; that is, the presence of electrolytes contracts the domain of $\Phi_{w}$ micellar region but the contraction is more in presence of strong electrolyte $(\mathrm{NaCl})$ than the weak one (oxalic acid). The presence of $\mathrm{NaCl}$, and oxalic acid has opposite effect on the one-phase micellar region. In the lower boundary of the phase diagram, presence of strong electrolyte, $\mathrm{NaCl}$ always decreases the domain of $1 \Phi$ micellar region whereas oxalic acid first decreases the domain $\Phi_{w}<$ 18 and then increases the temperature above $\Phi_{w}>18$. The critical weight fraction of water $\Phi_{w}{ }^{\text {cri }}$ decreases in presence of both electrolytes. Further, when cyclohexane is replaced by dodecane, the temperature window of $1 \Phi \mathrm{ME}$ region is tremendously increased.

\section{Acknowledgments}

The author thankfully acknowledges the financial support from Malaysian Ministry of Higher Education (MOHE) with Project no. FRGS59165.

\section{References}

[1] R. Belcher and L. Gordon, Kinetics of Precipitation, Pergamon Press, Oxford, UK, 1964.

[2] A. Bonazza, P. Brimblecombe, C. M. Grossi, and C. Sabbioni, "Carbon in black crusts from the Tower of London," Environmental Science and Technology, vol. 41, no. 12, pp. 4199-4204, 2007.

[3] L. Brecevic and D. Skrtic, "Transformation of calcium oxalate hydrates," Journal of Crystal Growth, vol. 74, no. 2, pp. 399408, 1986.

[4] H. H. M. Darweesh, "Building materials from siliceous clay and low grade dolomite rocks," Ceramics International, vol. 27, no. 1, pp. 45-50, 2001.

[5] B. L. Davis and G. Jixiang, "Airborne particulate study in five cities of China," Atmospheric Environment, vol. 34, no. 17, pp. 2703-2711, 2000.

[6] M. Z. H. Rozaini and P. Brimblecombe, "The solubility measurements of sodium dicarboxylate salts; sodium oxalate, malonate, succinate, glutarate, and adipate in water from $T=(279.15$ to 358.15$)$ K," Journal of Chemical Thermodynamic, vol. 41, no. 9, pp. 980-983, 2009.

[7] B. Ford, I. Macleod, and P. Haydock, "Rock art pigments from the Kimberly Region of Western Australia: iden-tification of the minerals and conversion mechanisms," Studies in Conservation, vol. 39, no. 1, pp. 57-69, 1994.

[8] J. H. Huang, Z. F. Mao, and M. F. Luo, "Effect of anionic surfactant on vaterite $\mathrm{CaCO}_{3}$," Materials Research Bulletin, vol. 42, no. 12, pp. 2184-2191, 2007.
[9] T. S. Jeong, J. H. Kim, M. S. Han, K. Y. Lim, and C. J. Youn, "Xray and cathodoluminescence study on the effect of intentional long time of succinic acid on calcium carbonate," Journal of Crystal Growth, vol. 280, no. 3-4, pp. 357-363, 2005.

[10] M. Z. H. Rozaini and P. Brimblecombe, "The odd-even behaviour of dicarboxylic acids solubility in the atmospheric aerosols," Water, Air, and Soil Pollution, vol. 198, no. 1-4, pp. 65-75, 2009.

[11] S. Mann, J. M. Didymus, N. P. Sanderson, B. R. Heywood, and E. J. A. Samper, "Morphological influence of functionalized and non-functionalized $\alpha, \omega$-dicarboxylates on calcite crystallization," Journal of the Chemical Society, Faraday Transactions, vol. 86, no. 10, pp. 1873-1880, 1990.

[12] M. G. S. Matteini, "The Protective effect of ammonium oxalate treatment on the surface of wall paintings," in Painted Facades. Proceedings of the Eurocare Project, pp. 95-101, Vienna, Austria, 1996.

[13] H. Okochi and P. Brimblecombe, "Potential trace metalorganic complexation in the atmosphere," The Scientific World Journal, vol. 2, pp. 767-786, 2002.

[14] C. Sabbioni and G. Zappia, "Atmospheric-derived element tracers on damaged stone," The Science of The Total Environment, vol. 126, no. 1-2, pp. 135-248, 1992.

[15] S. Signorelli, C. Peroni, M. Camaiti, and F. Fratini, "The presence of vaterite in bonding mortars of marble inlays from Florence Cathedral," Mineralogical Magazine, vol. 60, no. 4, pp. 663-665, 1996.

[16] O. Söhnel and J. W. Mullin, "Long-term and thermally stable superhydrophobic surfaces of carbon nanofibers," Journal of Colloid Interface Science, vol. 123, pp. 43-51, 1988.

[17] A.-W. Xu, M. Antonietti, H. Cölfen, and Y.-P. Fang, "Uniform hexagonal plates of vaterite $\mathrm{CaCO}_{3}$ mesocrystals formed by biomimetic mineralization," Advanced Functional Materials, vol. 16, no. 7, pp. 903-908, 2006.

[18] H. Wei, Q. Shen, Y. Zhao, D. J. Wang, and D. F. Xu, "Influence of polyvinylpyrrolidone on the precipitation of calcium carbonate and on the transformation of vaterite to calcite," Journal of Crystal Growth, vol. 250, no. 3-4, pp. 516-524, 2003.

[19] T. Kolev, B. Koleva, J. Kasperczyk et al., "Novel nonlinear optical materials based on dihydropyridine organic chromophore deposited on mica substrate," Journal of Materials Science: Materials in Electronics, vol. 20, no. 11, pp. 1073-1077, 2009. 

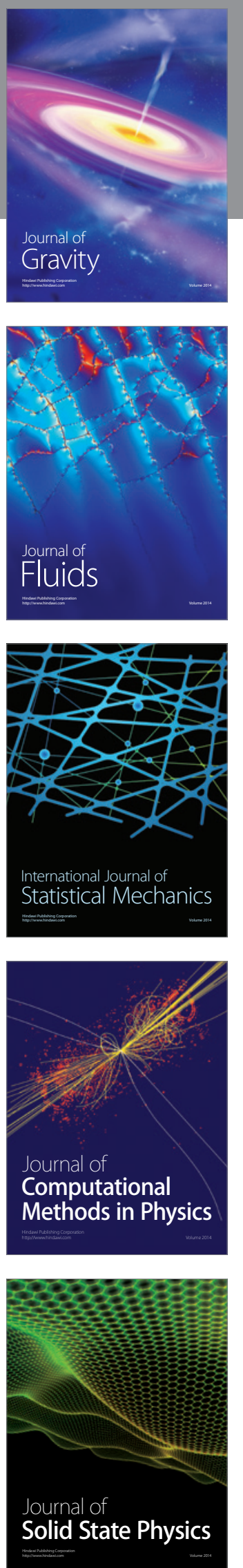

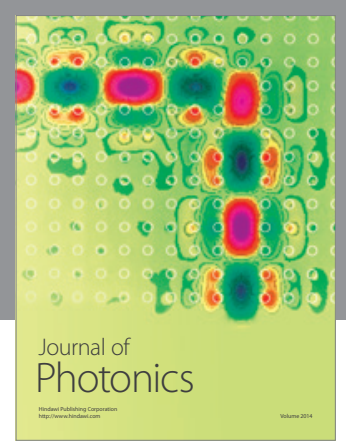

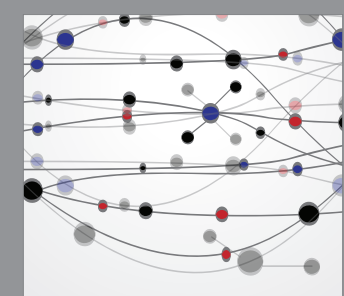

The Scientific World Journal
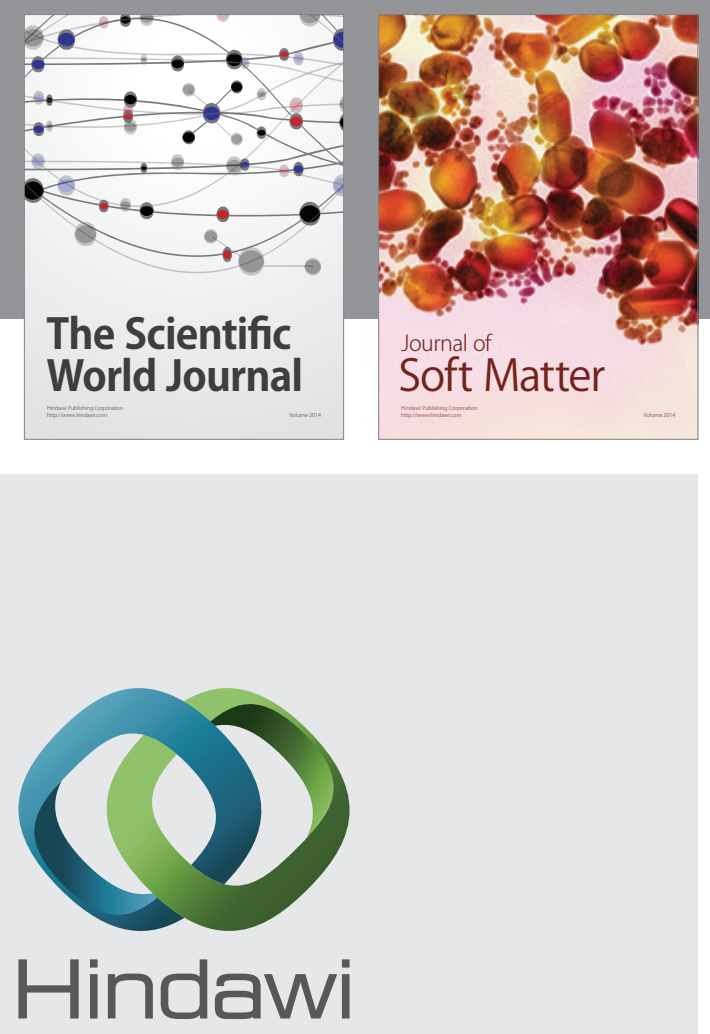

Submit your manuscripts at

http://www.hindawi.com
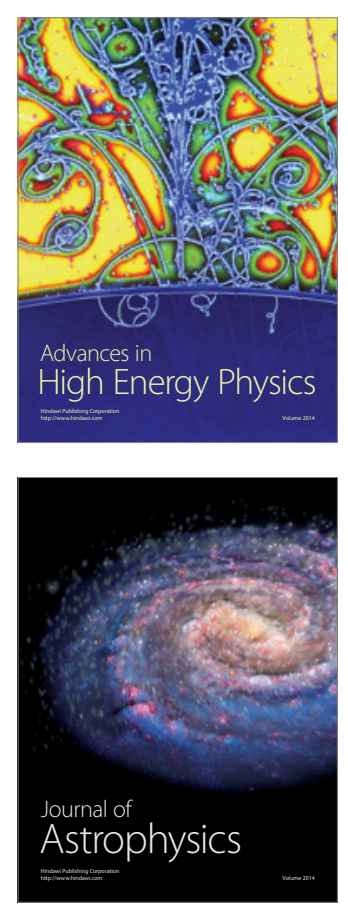
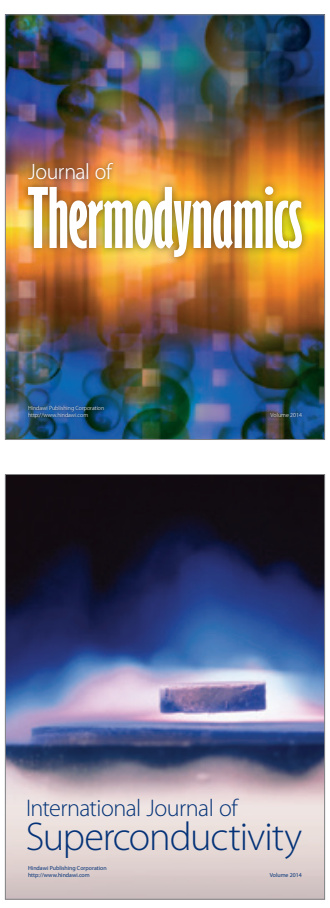
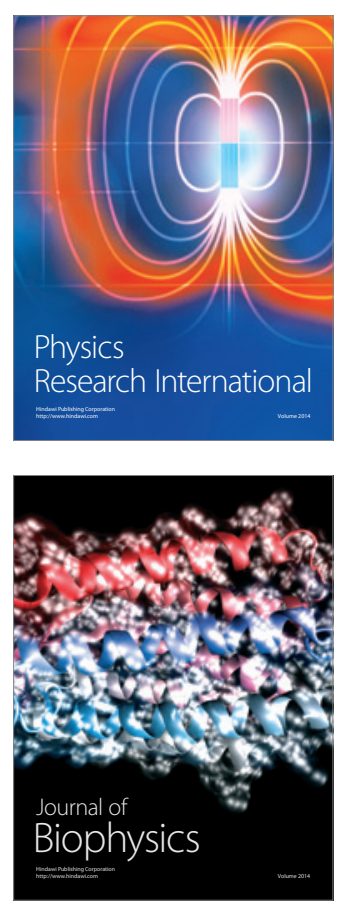
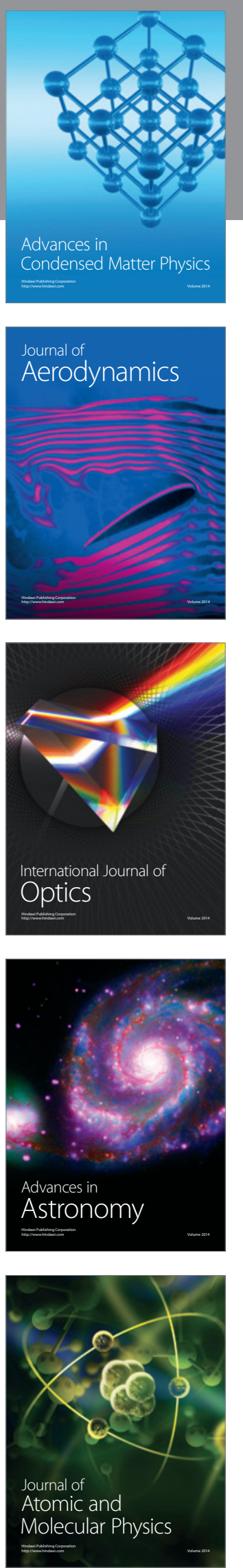